\title{
Predictors of intensive care unit admission in patients with Legionella pneumonia: role of the time to appropriate antibiotic therapy
}

\author{
Marco Falcone $^{1} \cdot$ Alessandro Russo $^{1} \cdot$ Giusy Tiseo $^{1} \cdot$ Mario Cesaretti $^{1} \cdot$ Fabio Guarracino $^{2} \cdot$ Francesco Menichetti $^{1}$
}

Received: 15 September 2020 / Accepted: 30 November 2020 / Published online: 14 December 2020

(c) The Author(s) 2020

\begin{abstract}
Purpose Legionella spp. pneumonia (LP) is a cause of community-acquired pneumonia (CAP) that requires early intervention. The median mortality rate varies from 4 to $11 \%$, but it is higher in patients admitted to intensive care unit (ICU). The objective of this study is to identify predictors of ICU admission in patients with LP.

Methods A single-center, retrospective, observational study conducted in an academic tertiary-care hospital in Pisa, Italy. Adult patients with LP consecutively admitted to study center from October 2012 to October 2019.

Results During the study period, 116 cases of LP were observed. The rate of ICU admission was $20.7 \%$ and the overall 30-day mortality rate was $12.1 \%$. Mortality was $4.3 \%$ in patients hospitalized in medical wards versus $41.7 \%$ in patients transferred to ICU $(p<0.001)$. The majority of patients $(74.1 \%)$ received levofloxacin as definitive therapy, followed by macrolides (16.4\%), and combination of levofloxacin plus a macrolide $(9.5 \%)$. In the multivariate analysis, diabetes (OR 8.28, CI 95\% 2.11-35.52, $p=0.002$ ), bilateral pneumonia (OR 10.1, CI 95\% 2.74-37.27, $p=0.001$ ), and cardiovascular events (OR 10.91, CI 95\% 2.83-42.01, $p=0.001$ ), were independently associated with ICU admission, while the receipt of macrolides/levofloxacin therapy within $24 \mathrm{~h}$ from admission was protective (OR 0.20, CI 95\% 0.05-0.73, $p=0.01$ ). Patients who received a late anti-Legionella antibiotic ( $>24 \mathrm{~h}$ from admission) underwent urinary antigen test later compared to those who received early active antibiotic therapy (2 [2-4] vs. $1[1-2]$ days, $p<0.001)$.

Conclusions Admission to ICU carries significantly increased mortality in patients with diagnosis of LP. Initial therapy with an antibiotic active against Legionella (levofloxacin or macrolides) reduces the probability to be transferred to ICU and should be provided in all cases until Legionella etiology is excluded.
\end{abstract}

Keywords Legionella $\cdot$ Intensive care unit · Pneumonia · Cardiovascular events · Empirical antibiotic therapy

\section{Introduction}

Legionella spp. is a causative agent in both sporadic and epidemic community-acquired pneumonia (CAP), but also in hospital outbreaks [1]. The introduction of urine antigen testing provides an early diagnosis of Legionella pneumonia (LP) in the majority of cases, reducing the risk of misdiagnosis and the delay in the administration of effective antibiotic therapy [2]. However, LP remains an infection associated

Marco Falcone

marco.falcone@unipi.it

1 Division of Infectious Diseases, Department of Clinical and Experimental Medicine, University of Pisa, Pisa, Italy

2 Department of Anaesthesia and Critical Care, Cardiothoracic and Vascular Anaesthesia and Intensive Care Medicine, Azienda Ospedaliero Universitaria Pisana, Pisa, Italy with substantial morbidity and mortality [3]. Intensive care unit (ICU) admission is recognized as an important risk factor for mortality, but unfortunately few data are reported about predictors of severe pneumonia and ICU admission in patients with confirmed LP [4].

The aim of this study was to identify factors independently associated with ICU admission in a series of consecutive patients affected by LP.

\section{Materials and methods}

This observational study was conducted between 2012 and 2019 on consecutive adult patients enrolled at the University hospital of Pisa, Italy. LP was diagnosed if two or more of the following were present: (1) rales, rhonchi, bronchial breath sounds, fever $\left(>38.0{ }^{\circ} \mathrm{C}\right)$, tachycardia, 
chills, dyspnea, coughing, chest pain; (2) presence of new consolidation(s) on chest X-ray; (3) diagnosis of Legionella spp. infection, defined by Legionella pneumophila serogroup 1 antigen in urine. The study was conducted according to the principles stated in the Declaration of Helsinki. The local Ethical Committee approved the study (approval number 1446).

Data on demographic characteristics, comorbidities, antibiotic, and concomitant therapy were retrospectively collected. Stratification of the severity of pneumonia at presentation was quantified by the Pneumonia Severity Index (PSI) and CURB-65 score [5]. CAP and hospital acquired pneumonia (HAP) were defined according to standard definitions [6]. Sepsis and septic shock were defined according to Sepsis-3 definition [7]. Cardiovascular event (CVE) included: (1) non-ST elevation myocardial infarction (NSTEMI); (2) ST elevation myocardial infarction (STEMI); (3) stroke; (4) a new episode of atrial fibrillation (AF); (5) deep venous thrombosis (DVT) and/or pulmonary embolism (PE); (6) new or worsening heart failure (HF); or (7) cardiovascular death [8].

Patients with pneumonia at the time of diagnosis underwent collection of blood cultures, detection of Legionella pneumophila serogroup 1 antigen in urine performed by immunochromatographic method (NOW Legionella Urinary Antigen Test; Binax Inc., Portland, ME), and culture of respiratory specimens.

To identify risk factors associated with the primary endpoint (ICU admission), univariate and multivariate analyses were performed. To detect significant differences between groups, we used the chi square test or Fisher's exact test for categorical variables and the two-tailed $t$ test or Mann-Whitney test for continuous variables, when appropriate. Continuous variables were reported as mean \pm standard deviation or median and interquartile ranges (IQRs) according to distribution; numbers and percentages were reported for categorical variables. Comparison of demographics, comorbidities and baseline variables (recorded at pneumonia onset) between patients who needed admission to ICU and those who did not was performed. Time from admission to receipt of anti-Legionella antibiotic (levofloxacin or macrolides) was dichotomized in two categories: $<=24 \mathrm{~h}$ and $>24 \mathrm{~h}$ from admission. All variables statistically significant at univariate analysis $(p<0.05)$ were included in the multivariate analysis to identify predictors of ICU admission. Multivariate analysis using logistic regression prediction models was constructed using a forward stepwise procedure; 95\% confidence intervals (CI) and odds ratios (OR) were calculated. Statistical significance was established at 0.05 . All reported $\mathrm{p}$ values are two-tailed.

The results obtained were analyzed using commercially available statistical software packages (SPSS, version 20.0; SPSS Inc, Chicago, Illinois).

\section{Results}

During the study period, 116 patients with LP were identified. Overall, 33 (28.4\%) underwent invasive or non-invasive mechanical ventilation, and $24(20.7 \%)$ were admitted to ICU. Thirty-day mortality was $12.1 \%$. An acute CVE was diagnosed in $24(20.7 \%)$ patients (12 new episodes of AF, 4 new or worsening HF, 4 DVT/PE, 2 NSTEMI, 1 STEMI and 1 cardiovascular death). The rate of ICU admission among patients who developed CVEs was $50 \%$.

As reported in Table 1, the 30-day mortality was $4.3 \%$ in patients admitted to medical wards compared to $41.7 \%$ in patients admitted to ICU $(p<0.001)$. Patients who needed ICU admission received an early (within $24 \mathrm{~h}$ from admission) antibiotic therapy including a drug covering Legionella less frequently compared to those who did not ( 54.2 vs. $80.4 \%, p=0.008$ ).

Overall, the median time from diagnosis of pneumonia to Legionella urinary test was 2 (IQRs 1-3) days. Patients admitted to ICU underwent Legionella urinary test later than those admitted to medical wards (2 [1-3] days versus 1 day [1-2], $p=0.042$ ). Patients treated with an antiLegionella antibiotic after $24 \mathrm{~h}$ from admission underwent urinary antigen test later than those who received active antibiotic therapy within $24 \mathrm{~h}(2[2-4]$ vs. 1 [1-2] days, $p<0.001$ ).

At the multivariate analysis (Table 2), diabetes (OR 8.28 , CI 95\% 2.11-35.52, $p=0.002$ ), bilateral pneumonia (OR 10.1, CI 95\% 2.74-37.27, $p=0.001$ ), and cardiovascular events (OR 10.91, CI 95\% 2.83-42.01, $p=0.001$ ), were independently associated with ICU admission, while receipt of macrolides/levofloxacin therapy within $24 \mathrm{~h}$ from admission was protective (OR 0.20, CI 95\% $0.05-0.73, p=0.01)$.

\section{Discussion}

Our data showed that some conditions such as diabetes, development of acute CVEs, and a bilateral involvement at chest radiograph are major predictors of ICU transfer. Furthermore, the administration of an initial (within $24 \mathrm{~h}$ from admission) antibiotic therapy covering Legionella is associated with reduced risk of ICU transfer. Thus, our study suggests that the initial antibiotic therapy of CAP should ever include a drug covering Legionella spp. until this etiology is excluded by microbiological tests.

A significant proportion of patients with LP require mechanical ventilation and admission to ICU (20.7\% and $28.4 \%$ of our patients, respectively). Thus, it is important to identify predictors of ICU admission when the patients 
Table 1 Univariate analysis of predictors of ICU admission in the study population

\begin{tabular}{|c|c|c|c|}
\hline Variables & $\begin{array}{l}\text { No ICU admission } \\
N=92(\%)\end{array}$ & $\begin{array}{l}\text { ICU admission } \\
N=24(\%)\end{array}$ & $p$ \\
\hline \multicolumn{4}{|l|}{ Baseline characteristics } \\
\hline Age, mean \pm SD & $71.2 \pm 14.2$ & $65.7 \pm 14.7$ & 0.97 \\
\hline Male sex & $59(64.1)$ & $17(70.8)$ & 0.634 \\
\hline CAP & $67(72.8)$ & $17(70.8)$ & 0.804 \\
\hline HAP & $5(5.4)$ & $3(12.5)$ & 0.359 \\
\hline Charlson comorbidity index, mean \pm SD & $3.3 \pm 2.1$ & $3.1 \pm 1.9$ & 0.78 \\
\hline Chronic heart disease & $12(13)$ & $3(12.5)$ & 1.0 \\
\hline Chronic liver disease & $3(3.3)$ & $3(12.5)$ & 0.102 \\
\hline Diabetes & $12(13)$ & $10(41.7)$ & 0.003 \\
\hline Neoplasm & $17(18.5)$ & $3(12.5)$ & 0.762 \\
\hline Chronic renal failure & $9(9.8)$ & $4(16.7)$ & 0.465 \\
\hline Hemodialysis & 0 & $2(8.3)$ & 0.041 \\
\hline COPD & $15(16.3)$ & $6(25)$ & 0.374 \\
\hline Immunocompromised status & $22(23.9)$ & $6(25)$ & 1.0 \\
\hline \multicolumn{4}{|l|}{ Clinical features and disease severity } \\
\hline Delirium at pneumonia onset & $36(39.1)$ & $12(50)$ & 0.36 \\
\hline Respiratory rate $\geq 30$ breaths/min & $5(5.4)$ & $8(33.3)$ & 0.001 \\
\hline Heart rate $\geq 125$ beats $/ \mathrm{min}$ & $13(14.1)$ & $11(45.8)$ & 0.002 \\
\hline Hypotension (MAP < 65 mmHg) & $8(8.7)$ & $6(25)$ & 0.04 \\
\hline Fever (body temperature $>37.5^{\circ} \mathrm{C}$ ) & $79(85.8)$ & $20(83.3)$ & 0.741 \\
\hline PSI class, mean \pm SD & $3.6 \pm 1$ & $4.1 \pm 0.8$ & 0.025 \\
\hline CURB-65, mean \pm SD & $1.3 \pm 0.8$ & $1.8 \pm 1$ & $\mathbf{0 . 0 2 7}$ \\
\hline Septic shock & $1(1.1)$ & $9(37.5)$ & $<0.001$ \\
\hline \multicolumn{4}{|l|}{ Radiological and laboratory findings } \\
\hline Pleural effusion & $33(35.9)$ & $16(66.7)$ & 0.01 \\
\hline Bilateral pneumonia & $23(25)$ & $16(66.7)$ & $<0.001$ \\
\hline Serum sodium $<130 \mathrm{mmol} / \mathrm{L}$ & $12(13)$ & $7(29.2)$ & $\mathbf{0 . 0 3 8}$ \\
\hline Leukocytosis (leukocytes $\geq 10.000 / \mu \mathrm{L}$ ) & $54(58.7)$ & $14(58.3)$ & 1.0 \\
\hline Leukopenia (leukocytes $<4.000 / \mu \mathrm{L}$ ) & $7(7.6)$ & $2(8.3)$ & 1.0 \\
\hline Hyperglycemia at pneumonia onset ( $>11 \mathrm{mmol} / \mathrm{L})$ & $4(4.3)$ & $5(20.8)$ & $\mathbf{0 . 0 1 5}$ \\
\hline Lactate $>2 \mathrm{mmol} / \mathrm{L}$ & $13(14.1)$ & $16(66.7)$ & $<0.001$ \\
\hline Platelets $<100.000 \mathrm{~mm}^{3}$ & $9(9.7)$ & $3(12.5)$ & 0.712 \\
\hline $\mathrm{LDH}(\mathrm{U} / \mathrm{L})$, mean $\pm \mathrm{SD}$ & $265.8 \pm 131.2$ & $465 \pm 247$ & $<0.001$ \\
\hline $\mathrm{PaO} 2 / \mathrm{FiO} 2$ ratio $<250$ & $27(29.3)$ & $14(58.3)$ & $\mathbf{0 . 0 3}$ \\
\hline \multicolumn{4}{|l|}{ Antimicrobial therapy } \\
\hline Levofloxacin therapy & $70(76.1 \%)$ & $15(62.5 \%)$ & 0.180 \\
\hline Macrolide therapy & $17(18.5 \%)$ & $5(20.8 \%)$ & 0.793 \\
\hline Macrolide + levofloxacin therapy & $5(5.4 \%)$ & $2(8.3 \%)$ & 0.595 \\
\hline Macrolide/levofloxacin therapy within $24 \mathrm{~h}$ from admission & $74(80.4 \%)$ & $13(54.2 \%)$ & $\mathbf{0 . 0 0 8}$ \\
\hline \multicolumn{4}{|l|}{ Outcomes } \\
\hline Mechanical invasive or non-invasive ventilation & $12(13)$ & $21(87.5)$ & $<0.001$ \\
\hline Cardiovascular events & $12(13)$ & $12(50)$ & $<0.001$ \\
\hline Median length of hospitalization, mean days $\pm \mathrm{SD}$ & $8.3 \pm 3.7$ & $26.1 \pm 19.2$ & $<0.001$ \\
\hline 30-day mortality & $4(4.3)$ & $10(41.7)$ & $<0.001$ \\
\hline
\end{tabular}

$I C U$ intensive care unit, $S D$ standard deviation, $C A P$ community-acquired pneumonia, HCAP healthcare-associated pneumonia, $H A P$ hospitalacquired pneumonia, COPD chronic obstructive pulmonary disease, $H I V$ human immunodeficiency virus, $L D H$ lactic acid dehydrogenase, $P S I$ pneumonia severity index, $A R D S$ acute respiratory distress syndrome

Bold values indicate statistical significance $(p<0.05)$ 
Table 2 Multivariate analysis of predictors of ICU admission in the study population

\begin{tabular}{lcll}
\hline Variables & OR & $95 \%$ CI & $p$ \\
\hline Cardiovascular events & 10.91 & $2.83-42.01$ & 0.001 \\
Bilateral pneumonia & 10.1 & $2.74-37.27$ & 0.001 \\
Diabetes & 8.28 & $2.11-35.52$ & 0.002 \\
$\begin{array}{l}\text { Macrolides/levofloxacin therapy } \\
\text { within 24 h from admission }\end{array}$ & 0.20 & $0.05-0.73$ & 0.01 \\
\hline
\end{tabular}

$I C U$ intensive care unit, $O R$ odds ratio, $C I$ Confidence intervals

arrive at the Emergency Department. Remarkably, we found a strong association with diabetes and development of CVEs. Diabetes is a recognized risk factor for early and late mortality in patients with CAP [9], and is a condition frequently associated with the development of acute CVEs $[10,11])$. Most CVEs occur in patients with underlying cardiovascular disease [8]. These data suggest that among patients with severe LP a careful evaluation of cardiovascular parameters should be performed at admission and during the hospital stay: among patients with risk factors for CVE, such as diabetic ones, Troponin and BNP levels should be measured and a strict surveillance, with the use also of cardiovascular ultrasound, is needed to recognize and to treat acute CVEs that may be fatal for the patient.

On the other hand, we found that the administration of an early antibiotic regimen containing at least one drug active against Legionella (macrolides or levofloxacin) was a factor significantly associated with reduced risk of ICU admission. We also noted that urinary antigen test for Legionella was not performed at hospital arrival in all cases, but after a median of 2 days. A delay in the administration of antibiotics active against Legionella was then directly correlated to a delay in the performance of urinary antigen test. These findings lead to some important considerations: first, it is mandatory to perform urinary antigen test for Legionella at the time of hospital admission in all cases of severe CAP; this is a recommendation already contained in the current guidelines for CAP [12]. Second, if the test is not available at the time of CAP diagnosis, initial antimicrobial therapy should include a drug with in vitro activity against Legionella (azithromycin, clarithromycin, or levofloxacin); to this end, a recent systematic review found no difference in the effectiveness of fluoroquinolones vs. macrolides in reducing mortality among patients with LP [13], although very few data compared the two classes of antibiotics in some settings such as immunosuppressed patients, severe patients needing ICU admission and patients with nosocomial legionellosis [14]. Finally, a positive or negative urinary antigen test prompts withdrawal or continuation of antibiotic treatment directed at Legionella pathogen. This strategy improves the antimicrobial prescriptions, by reducing the number of unnecessary antibiotics.

Of importance, no serious adverse events (AEs) directly related to levofloxacin/macrolides administration were recorded in our study population. However, in patients with preexisting cardiovascular diseases an evaluation of the possible AEs should be carefully performed before starting therapy [15].

Our study has several limitations. First, the retrospective design of the study is an intrinsic limitation; second, the relative small sample size does not permit to obtain definite conclusions. Anyway, considering that Legionella is an uncommon etiology of pneumonia, our series is one of the largest published in the last years; finally, as regard to decisions adopted in patients at the end of life, in particular "do not resuscitate order" and preclusion to invasive ventilation, there was not a pre-defined protocol and the decision was taken by the physician in charge according to the individual decision and conditions of each patient. It is also important to underline that the urinary antigen test (that includes only Legionella serogroup 1) has low sensitivity: a recent paper reported that, compared to PCR molecular test, urinary test results appeared false negative in the $44.4 \%$ of cases of LP and a total of $39.4 \%$ (26/66) diagnosis probably would have been missed or delayed without a syndromic approach [16]. Thus, physicians should consider to treat cases with a high probability of LP but negative urinary test [17]. Future prospective studies will clarify this point.

In conclusion, the knowledge of predictors for ICU admission could stimulate future studies to understand what therapeutic approaches should be more useful in criticallyill patients, to reduce unfavorable outcomes like CVEs that are associated with increased risk of ICU admission and progression to death.

Author contributions Conceived and designed study: MF, AR, GT, FM. Performed data collection: MC. Analyzed data: AR, GT. Wrote the paper: MF, AR, GT, FM.

Funding Open access funding provided by Università di Pisa within the CRUI-CARE Agreement. None to declare.

\section{Compliance with ethical standards}

\section{Conflict of interest None to declare.}

Open Access This article is licensed under a Creative Commons Attribution 4.0 International License, which permits use, sharing, adaptation, distribution and reproduction in any medium or format, as long as you give appropriate credit to the original author(s) and the source, provide a link to the Creative Commons licence, and indicate if changes were made. The images or other third party material in this article are included in the article's Creative Commons licence, unless indicated otherwise in a credit line to the material. If material is not included in the article's Creative Commons licence and your intended use is not 
permitted by statutory regulation or exceeds the permitted use, you will need to obtain permission directly from the copyright holder. To view a copy of this licence, visit http://creativecommons.org/licenses/by/4.0/.

\section{References}

1. Herwaldt LA, Marra AR. Legionella: a reemerging pathogen. Curr Opin Infect Dis. 2018;31:325-33.

2. Blàzquez Garrido RM, Espinosa Parra FJ, Alemany Francè L, et al. Antimicrobial chemotherapy for Legionnaires' disease: levofloxacin versus macrolides. Clin Infect Dis. 2005;40:800e6.

3. Mykietiuk A, Carratalà J, Fernàndez-Sabè N, et al. Clinical outcomes for hospitalized patients with Legionella pneumonia in the antigenuria. Clin Infect Dis. 2005;40:794-9.

4. Arancibia F, Cortes CP, Valdés M, et al. Importance of Legionella pneumophila in the etiology of severe community-acquired pneumonia in Santiago. Chile Chest. 2014;145:290-6.

5. Falcone M, Corrao S, Venditti M, Serra P, Licata G. Performance of PSI, CURB-65, and SCAP scores in predicting the outcome of patients with community-acquired and healthcare-associated pneumonia. Intern Emerg Med. 2011;6:431-6.

6. Falcone M, Russo A, Giannella M, et al. Individualizing risk of multidrug-resistant pathogens in community-onset pneumonia. PLoS ONE. 2015;10:e0119528.

7. Singer M, Deutschman CS, Seymour CW, et al. The third International consensus definitions for sepsis and septic shock (Sepsis-3). JAMA. 2016;315:801-10.

8. Violi F, Cangemi R, Falcone M, et al. Cardiovascular complications and short-term mortality risk in community-acquired pneumonia. Clin Infect Dis. 2017;64:1486-93.

9. Falcone M, Tiseo G, Russo A, et al. Hospitalization for pneumonia is associated with decreased 1-year survival in patients with type 2 diabetes: results from a prospective cohort study. Medicine (Baltimore). 2016;95:e2531.

10. Falcone M, Russo A, Cangemi R, et al. Lower mortality rate in elderly patients with community-onset pneumonia on treatment with aspirin. J Am Heart Assoc. 2015;4(1):e001595.

11. Violi F, Carnevale R, Calvieri C, et al. Nox2 up-regulation is associated with an enhanced risk of atrial fibrillation in patients with pneumonia. Thorax. 2015;70:961-6.

12. Metlay JP, Waterer GW, Long AC, et al. Diagnosis and treatment of adults with community-acquired Pneumonia. An official clinical practice guideline of the American Thoracic Society and Infectious Diseases Society of America. Am J Respir Crit Care Med. 2019;200:e45-67.

13. Jasper AS, Musuuza JS, Tischendorf JS, Stevens VW, Gamage $\mathrm{SD}$, Osman F, et al. Are fluoroquinolones or macrolides better for treating Legionella pneumonia? A systematic review and metaanalysis. Clin Infect Dis. 2020. https://doi.org/10.1093/cid/ciaa4 41.

14. Torres A, Cillóniz C. Are macrolides as effective as fluoroquinolones in Legionella pneumonia? YES, But.... Clin Infect Dis. 2020. https://doi.org/10.1093/cid/ciaa442 (Online ahead of print).

15. König R, Cao X, Oswald M, et al. Macrolide combination therapy for patients hospitalised with community-acquired pneumonia? An individualised approach supported by machine learning. Eur Respir J. 2019;54:1900824.

16. Muyldermans A, Descheemaeker P, Boel A, et al. What is the risk of missing legionellosis relying on urinary antigen testing solely? A retrospective Belgian multicenter study. Eur J Clin Microbiol Infect Dis. 2020;39:729-34.

17. Waterer GW, Baselski VS, Wunderink RG. Legionella and community-acquired pneumonia: a review of current diagnostic tests from a clinician's viewpoint. Am J Med. 2001;110:41-8. 